\title{
CONTACT STRUCTURE ON MIXED LINKS
}

\author{
MUTSUO OKA
}

\begin{abstract}
A strongly non-degenerate mixed function has a Milnor open book structures on a sufficiently small sphere. We introduce the notion of a holomorphic-like mixed function and we will show that a link defined by such a mixed function has a canonical contact structure. Then we will show that this contact structure for a certain holomorphiclike mixed function is carried by the Milnor open book.
\end{abstract}

\section{INTRODUCTION}

Let $f(\mathbf{z})$ a holomorphic function with an isolated critical point at the origin. Then the Milnor fibration of $f$ carries a canonical contact structure $([4,2])$. We consider a similar problem for mixed functions $f(\mathbf{z}, \overline{\mathbf{z}})$. We have shown that strongly non-degenerate mixed functions have Milnor fibrations on a small sphere [9]. However the situation is very different in the point that the tangent space of a mixed hypersurface is not a complex vector space. Therefore the restriction of the canonical contact structure need not give a contact structure on the mixed link. We introduce a class of mixed functions called holomorphic-like and we show that the restriction of the canonical contact structure gives a contact structure on the link (Theorem 13). A typical class of mixed functions we consider are given as the pullback $g(\mathbf{w}, \overline{\mathbf{w}})=\varphi_{a, b}^{*} f(\mathbf{w}, \overline{\mathbf{w}})$ of a convenient non-degenerate holomorphic function $f(\mathbf{z})$ by a homogeneous mixed covering $\varphi_{a, b}: \mathbb{C}^{n} \rightarrow \mathbb{C}^{n}$ which is defined by $\varphi_{a, b}(\mathbf{w}, \overline{\mathbf{w}})=\left(w_{1}^{a} \bar{w}_{1}^{b}, \ldots, w_{n}^{a} \bar{w}_{n}^{b}\right)$. Such a pull-back is a typical example of a holomorphic-like mixed function. Then we will show also that the Milnor open book is compatible with the canonical contact structures for these mixed functions (Theorem 22).

I would like to thank M. Ishikawa and V. Blanloeil for the stimulating discussions.

\section{Preliminaries}

2.1. Mixed functions and polar weightedness. Consider complex analytic function of $2 n$-variables $F\left(z_{1}, \ldots, z_{n}, w_{1}, \ldots, w_{n}\right)$ expanded in a convergent series $\sum_{\nu, \mu} c_{\nu, \mu} \mathbf{z}^{\nu} \mathbf{w}^{\mu}$ and consider the restriction $f(\mathbf{z}, \overline{\mathbf{z}})$ which is defined by the substitution $w_{j}=\bar{z}_{j}, j=1, \ldots, n$. We call this real analytic function an analytic mixed function. Namely $f(\mathbf{z}, \overline{\mathbf{z}})=\sum_{\nu, \mu} c_{\nu, \mu} \mathbf{z}^{\nu} \overline{\mathbf{z}}^{\mu}$

2000 Mathematics Subject Classification. 32S55,53D10,32S25.

Key words and phrases. Contact structure, Milnor fibration, holomophic-like. 
where $\mathbf{z}=\left(z_{1}, \ldots, z_{n}\right), \overline{\mathbf{z}}=\left(\bar{z}_{1}, \ldots, \bar{z}_{n}\right), \mathbf{z}^{\nu}=z_{1}^{\nu_{1}} \cdots z_{n}^{\nu_{n}}$ for $\nu=\left(\nu_{1}, \ldots, \nu_{n}\right)$ (respectively $\overline{\mathbf{z}}^{\mu}=\bar{z}_{1}^{\mu_{1}} \cdots \bar{z}_{n}^{\mu_{n}}$ for $\mu=\left(\mu_{1}, \ldots, \mu_{n}\right)$ ). Here $\bar{z}_{i}$ is the complex conjugate of $z_{i}$. Assume that $f$ is a polynomial. Writing $z_{j}=x_{j}+i y_{j}$, it is easy to see that $f$ is a polynomial of $2 n$-variables $x_{1}, y_{1}, \ldots, x_{n}, y_{n}$. In this case, we call $f$ a mixed polynomial of $z_{1}, \ldots, z_{n}$.

A mixed polynomial $f(\mathbf{z}, \overline{\mathbf{z}})$ is called polar weighted homogeneous if there exist positive integers $q_{1}, \ldots, q_{n}$ and $p_{1}, \ldots, p_{n}$ and non-zero integers $m_{r}, m_{p}$ such that

$$
\begin{gathered}
\operatorname{gcd}\left(q_{1}, \ldots, q_{n}\right)=1, \quad \operatorname{gcd}\left(p_{1}, \ldots, p_{n}\right)=1, \\
\sum_{j=1}^{n} q_{j}\left(\nu_{j}+\mu_{j}\right)=m_{r}, \quad \sum_{j=1}^{n} p_{j}\left(\nu_{j}-\mu_{j}\right)=m_{p}, \quad \text { if } c_{\nu, \mu} \neq 0
\end{gathered}
$$

The weight vectors $Q=\left(q_{1}, \ldots, q_{n}\right)$ and $P=\left(p_{1}, \ldots, p_{n}\right)$ are called the radial weight and the polar weight respectively. Using radial weight and the polar weight, we define the radial $\mathbb{R}_{>0}$-action and the polar $S^{1}$-action as follows.

$$
\begin{aligned}
r \circ \mathbf{z}=\left(r^{q_{1}} z_{1}, \ldots, r^{q_{n}} z_{n}\right), & r \in \mathbb{R}_{>0} \\
e^{i \eta} \circ \mathbf{z} \quad & =\left(e^{i p_{1} \eta} z_{1}, \ldots, e^{i p_{n} \eta} z_{n}\right), \quad e^{i \eta} \in S^{1}
\end{aligned}
$$

Then $f$ satisfies the functional equalities

$$
\begin{array}{r}
f(r \circ(\mathbf{z}, \overline{\mathbf{z}})) \quad=r^{m_{r}} f(\mathbf{z}, \overline{\mathbf{z}}), \quad r \in \mathbb{R}_{>0} \\
f\left(e^{i \eta} \circ(\mathbf{z}, \overline{\mathbf{z}})\right) \quad=e^{i m_{p} \eta} f(\mathbf{z}, \overline{\mathbf{z}}), e^{i \eta} \in S^{1} .
\end{array}
$$

These equalities give the following Euler equalities.

$$
\begin{aligned}
& \text { (Radial Euler equality) }: m_{r} f(\mathbf{z}, \overline{\mathbf{z}})=\sum_{i=1}^{n} q_{i}\left(\frac{\partial f}{\partial z_{i}} z_{i}+\frac{\partial f}{\partial \bar{z}_{i}} \bar{z}_{i}\right) \\
& \text { (Polar Euler equality) }: m_{p} f(\mathbf{z}, \overline{\mathbf{z}})=\sum_{i=1}^{n} p_{i}\left(\frac{\partial f}{\partial z_{i}} z_{i}-\frac{\partial f}{\partial \bar{z}_{i}} \bar{z}_{i}\right) .
\end{aligned}
$$

We consider a special type of polar weighted homogeneous polynomial. A polar weighted homogeneous polynomial $f(\mathbf{z}, \overline{\mathbf{z}})$ is called strongly polar weighted homogeneous if the radial weight and the polar weights are the same, i.e., $p_{j}=q_{j}$ for $j=1, \ldots, n$. In this case, the radial and polar Euler equalities gives:

$$
\left\{\begin{array}{l}
\sum_{j=1}^{n} p_{j} z_{j} \frac{\partial f}{\partial z_{j}}(\mathbf{z}, \overline{\mathbf{z}})=\frac{m_{r}+m_{p}}{2} f(\mathbf{z}, \overline{\mathbf{z}}), \\
\sum_{j=1}^{n} p_{j} \bar{z}_{j} \frac{\partial f}{\partial \bar{z}_{j}}(\mathbf{z}, \overline{\mathbf{z}})=\frac{m_{r}-m_{p}}{2} f(\mathbf{z}, \overline{\mathbf{z}})
\end{array}\right.
$$

The above equalities say that $f(\mathbf{z}, \overline{\mathbf{z}})$ is a weighted homogeneous polynomial for $\mathbf{z}$ and $\overline{\mathbf{z}}$ independently. Furthermore $f(\mathbf{z}, \overline{\mathbf{z}})$ is called strongly polar positive weighted homogeneous if $\operatorname{pdeg} f=m_{p}>0$.

2.2. Euclidean metric and hermitian product. Recall that $\mathbb{C}^{n}$ is canonically identified with $\mathbb{R}^{2 n}$ by $\mathbf{z}=\left(z_{1}, \ldots, z_{n}\right) \mapsto \mathbf{z}_{\mathbb{R}}:=\left(x_{1}, y_{1}, \ldots, x_{n}, y_{n}\right) \in$ $\mathbb{R}^{2 n}$. The inner product in $\mathbb{R}^{2 n}$ is simply the real part of the hermitian product in $\mathbb{C}^{n}$. We denote the hermitian inner product as $(\mathbf{z}, \mathbf{w})$ for $\mathbf{z}, \mathbf{w} \in \mathbb{C}^{n}$ 
and the inner product as the vector in $\mathbb{R}^{2 n}$ as $\left(\mathbf{z}_{\mathbb{R}}, \mathbf{w}_{\mathbb{R}}\right)_{\mathbb{R}}$. Namely putting $\mathbf{w}=\left(w_{1}, \ldots, w_{n}\right)$ with $w_{j}=u_{j}+i v_{j}$,

$$
(\mathbf{z}, \mathbf{w})=\sum_{j=1}^{n} z_{j} \bar{w}_{j}, \quad\left(\mathbf{z}_{\mathbb{R}}, \mathbf{w}_{\mathbb{R}}\right)_{\mathbb{R}}=\sum_{j=1}^{n}\left(x_{j} u_{j}+y_{j} v_{j}\right) .
$$

Thus $\Re(\mathbf{z}, \mathbf{w})=\left(\mathbf{z}_{\mathbb{R}}, \mathbf{w}_{\mathbb{R}}\right)_{\mathbb{R}}$. By the triviality of the tangent bundle $T_{p} \mathbb{R}^{2 n}$, we identify $T_{p} \mathbb{R}^{2 n}$ with $\mathbb{R}^{2 n}=\mathbb{C}^{n}$. Thus $\sum_{j=1}^{n}\left(x_{j}\left(\frac{\partial}{\partial x_{j}}\right)_{p}+y_{j}\left(\frac{\partial}{\partial y_{j}}\right)_{p}\right)$ is identified with $\mathbf{z}=\left(z_{1}, \ldots, z_{n}\right) \in \mathbb{C}^{n}$ and $z_{j}=x_{j}+i y_{j}$. Recall the complexified tangent vectors are defined by

$$
\frac{\partial}{\partial z_{j}}=\frac{1}{2}\left(\frac{\partial}{\partial x_{j}}-i \frac{\partial}{\partial y_{j}}\right), \quad \frac{\partial}{\partial \bar{z}_{j}}=\frac{1}{2}\left(\frac{\partial}{\partial x_{j}}+i \frac{\partial}{\partial y_{j}}\right)
$$

Thus a complex vector $\mathbf{z} \in \mathbb{C}^{2 n}=\mathbb{R}^{2 n}$ is identified with the tangent vector

$$
\sum_{j=1}^{n}\left(x_{j} \frac{\partial}{\partial x_{j}}+y_{j} \frac{\partial}{\partial y_{j}}\right)=\sum_{j=1}^{n}\left(z_{j} \frac{\partial}{\partial z_{j}}+\bar{z}_{j} \frac{\partial}{\partial \bar{z}_{j}}\right) .
$$

$J: T \mathbb{C}^{n} \rightarrow T \mathbb{C}^{n}$ is the almost complex structure defined by

$$
\begin{gathered}
J\left(\frac{\partial}{\partial x_{j}}\right)=\frac{\partial}{\partial y_{j}}, \quad J\left(\frac{\partial}{\partial y_{j}}\right)=-\frac{\partial}{\partial x_{j}} \\
J\left(\frac{\partial}{\partial z_{j}}\right)=i \frac{\partial}{\partial z_{j}}, \quad J\left(\frac{\partial}{\partial \bar{z}_{j}}\right)=-i \frac{\partial}{\partial \bar{z}_{j}} .
\end{gathered}
$$

For a real valued mixed function $h(\mathbf{z}, \overline{\mathbf{z}})$, we define the real gradient $\operatorname{grad}_{\mathbb{R}} \mathrm{h} \in$ $\mathbb{R}^{2 \mathrm{n}}$ (or Riemannian gradient in [2]) as

$$
\operatorname{grad}_{\mathbb{R}} h(\mathbf{z}, \overline{\mathbf{z}})=\left(\frac{\partial h}{\partial x_{1}}(\mathbf{z}, \overline{\mathbf{z}}), \frac{\partial h}{\partial y_{1}}(\mathbf{z}, \overline{\mathbf{z}}) \ldots, \frac{\partial h}{\partial x_{n}}(\mathbf{z}, \overline{\mathbf{z}}), \frac{\partial h}{\partial y_{n}}(\mathbf{z}, \overline{\mathbf{z}})\right) \in \mathbb{R}^{2 n} .
$$

We define also the complex gradient of $h$ or hermitian gradient in [2] as follows.

$$
\nabla h(\mathbf{z}, \overline{\mathbf{z}})=2\left(\frac{\overline{\partial h}}{\partial z_{1}}(\mathbf{z}, \overline{\mathbf{z}}), \ldots, \frac{\overline{\partial h}}{\partial z_{n}}(\mathbf{z}, \overline{\mathbf{z}})\right) \in \mathbb{C}^{n} .
$$

Proposition 1. Assume that $h(\mathbf{z}, \overline{\mathbf{z}})$ is a real valued mixed function and let $\mathbf{z}(t)=\left(z_{1}(t), \ldots, z_{n}(t)\right), z_{j}(t)=x_{j}(t)+i y_{j}(t),-1 \leq t \leq 1$ be a smooth curve in $\mathbb{C}^{n}$ and let $\mathbf{z}(0)=\mathbf{u}, \frac{d \mathbf{z}}{d t}(0)=\mathbf{v}$. Then we have

$$
\frac{d h(\mathbf{z}(t), \overline{\mathbf{z}}(t))}{d t}(0)=\left(\mathbf{v}_{\mathbb{R}}, \operatorname{grad}_{\mathbb{R}} \mathrm{h}(\mathbf{u}, \overline{\mathbf{u}})\right)_{\mathbb{R}}=\Re(\mathbf{v}, \nabla h(\mathbf{u}, \overline{\mathbf{u}})) .
$$

Proof. The second equality follows from the simple calculation:

$$
\begin{aligned}
\frac{d h(\mathbf{z}(t), \overline{\mathbf{z}}(t))}{d t}(0) & =\sum_{i=1}^{n} v_{i} \frac{\partial h}{\partial z_{i}}\left(\mathbf{z}_{0}, \overline{\mathbf{z}}_{0}\right)+\sum_{i=1}^{n} \bar{v}_{i} \frac{\partial h}{\partial \bar{z}_{i}}\left(\mathbf{z}_{0}, \overline{\mathbf{z}}_{0}\right) \\
& =\sum_{i=1}^{n} v_{i} \frac{\partial h}{\partial z_{i}}\left(\mathbf{z}_{0}, \overline{\mathbf{z}}_{0}\right)+\sum_{i=1}^{n} \bar{v}_{i} \frac{\overline{\partial h}}{\partial z_{i}}\left(\mathbf{z}_{0}, \overline{\mathbf{z}}_{0}\right) \\
& =\Re\left(\mathbf{v}, \nabla h\left(\mathbf{z}_{0}, \overline{\mathbf{z}}_{0}\right)\right) .
\end{aligned}
$$


Thus the tangent space of the real hypersurface $H:=h^{-1}(0)$ at a smooth point $\mathbf{z}_{0}$ is given by

$$
\begin{aligned}
T_{\mathbf{X}_{0}} H & =\left\{\mathbf{u} \in \mathbb{R}^{2 n} \mid\left(\mathbf{u}, \operatorname{grad}_{\mathbb{R}} h\left(\mathbf{z}_{0}, \overline{\mathbf{z}}_{0}\right)\right)_{\mathbb{R}}=0\right\} \\
& =\left\{\mathbf{w} \in \mathbb{C}^{n} \mid \Re\left(\mathbf{w}, \nabla h\left(\mathbf{z}_{0}, \overline{\mathbf{z}}_{0}\right)\right)=0\right\} .
\end{aligned}
$$

For our later purpose, it is more convenient to use the hermitian gradient.

2.2.1. Holomorphic function case. Assume that $f(\mathbf{z})$ is a holomorphic function defined on a neighborhood of the origin. Then the hermitian gradient $\nabla f$ is defined by (see [6, 2] )

$$
\nabla f(\mathbf{z})=\left(\overline{\frac{\partial f}{\partial z_{1}}}, \ldots, \overline{\frac{\partial f}{\partial z_{n}}}\right) .
$$

Consider the complex hypersurface $V=f^{-1}(0) \subset \mathbb{C}^{n}$.

Proposition 2. Assume that $p \in V$ is a regular point. Then the tangent space $T_{p} V$ is the complex subspace given by

$$
T_{p} V=\left\{\mathbf{v} \in \mathbb{C}^{n} \mid(\mathbf{v}, \nabla f(\mathbf{z}))=0\right\} .
$$

Remark 3. Let $f(\mathbf{z}, \overline{\mathbf{z}})$ be a complex valued mixed function and write $f(\mathbf{z}, \overline{\mathbf{z}})=$ $g(\mathbf{z}, \overline{\mathbf{z}})+i h(\mathbf{z}, \overline{\mathbf{z}})$. Consider a mixed hypersurface $V=f^{-1}(0)$ and assume that $p \in V$ is a mixed regular point. Then the tangent space $T_{p} V$ has no complex structure in general and there does not exist a single gradient vector to describe $T_{p} V$. It is described by two hermitian gradient vectors as

$$
T_{p} V=\left\{\mathbf{v} \in \mathbb{C}^{n} \mid \Re(\mathbf{v}, \nabla g(p, \bar{p}))=\Re(\mathbf{v}, \nabla h(p, \bar{p}))=0\right\} .
$$

2.2.2. Weighted spheres. For a given positive integer vector $\mathbf{a}=\left(a_{1}, \ldots, a_{n}\right)$ and a positive number $r>0$, we consider

$$
\rho_{\mathbf{a}}(\mathbf{z})=\sum_{j=1}^{n} a_{j}\left|z_{j}\right|^{2}=\sum_{j=1}^{n} a_{j}\left(x_{j}^{2}+y_{j}^{2}\right)
$$

and we define the weighted sphere $\mathbb{S}_{r}(\mathbf{a})$ by

$$
\mathbb{S}_{r}(\mathbf{a}):=\left\{\mathbf{z} \in \mathbb{C}^{n} \mid \rho_{\mathbf{a}}(\mathbf{z})=r^{2}\right\}
$$

The standard sphere is defined by the weight vector $\mathbf{a}=(1, \ldots, 1)$ and in this case, we simply write $\mathbb{S}_{r}$. Put $\tilde{\mathbf{z}}(\mathbf{a})=\left(a_{1} z_{1}, \ldots, a_{n} z_{n}\right)$. Then $\nabla \rho \mathbf{a}(\mathbf{z})=$ $2 \tilde{\mathbf{z}}(\mathbf{a})$. Therefore the tangent space at $\mathbf{z}_{0} \in \mathbb{S}_{r}(\mathbf{a})$ is given by

$$
T_{\mathbf{Z}_{0}} \mathbb{S}_{r}(\mathbf{a})=\left\{\mathbf{w} \mid \Re\left(\mathbf{w}, \tilde{\mathbf{z}}_{0}(\mathbf{a})\right)=0\right\}, \quad T_{\mathbf{z}_{0}} \mathbb{S}_{r}=\left\{\mathbf{w} \mid \Re\left(\mathbf{w}, \mathbf{z}_{0}\right)=0\right\} .
$$


2.2.3. Transversality of a polar weighted homogeneous hypersurface. Let $f$ be a polar weighted homogeneous polynomial of radial weight type

$\left(q_{1}, \ldots, q_{n} ; m_{r}\right)$ and of polar weight type $\left(p_{1}, \ldots, p_{n} ; m_{p}\right)$. Let $V=f^{-1}(0)$ and write $f(\mathbf{z}, \overline{\mathbf{z}})=h(\mathbf{z}, \overline{\mathbf{z}})+i g(\mathbf{z}, \overline{\mathbf{z}})$ with real valued mixed functions $h, g$.

Proposition 4. (Transversality) Assume that $V$ has an isolated mixed singularity at the origin. Then the sphere $\mathbb{S}_{r}(\mathbf{a})=\left\{\mathbf{z} \in \mathbb{C}^{n} ; \rho \mathbf{a}(\mathbf{z})=r^{2}\right\}$ intersects transversely with $V$ for any $r>0$.

Proof. The proof is the exact same as that of Proposition 4, 8]. Assume that $\mathbf{z}_{0} \in S_{\mathbf{a}}(r) \cap V$ is a point where the sphere is not transverse. Note that the tangent space is the real orthogonal space to two hermitian gradient vectors $\nabla h\left(\mathbf{z}_{0}, \overline{\mathbf{z}}_{0}\right)$ and $\nabla g\left(\mathbf{z}_{0}, \overline{\mathbf{z}}_{0}\right)$. Let $\rho \mathbf{a}(\mathbf{z}, \overline{\mathbf{z}})=\sum_{j=1}^{n} a_{j}\left|z_{j}\right|^{2}$. The nontransversality implies for example, there is a linear relation

$$
\nabla \rho \mathbf{a}\left(\mathbf{z}_{0}, \overline{\mathbf{z}}_{0}\right)=\alpha \nabla h\left(\mathbf{z}_{0}, \overline{\mathbf{z}}_{0}\right)+\beta \nabla g\left(\mathbf{z}_{0}, \overline{\mathbf{z}}_{0}\right)
$$

with some $\alpha, \beta \in \mathbb{R}$. We consider the radial orbit curve $\mathbf{z}(t)=t \circ \mathbf{z}_{0}=$ $\left(t^{q_{1}} z_{01}, \ldots, t^{q_{n}} z_{0 n}\right)$. The the tangent vector $\frac{d z}{d t}(1)=\tilde{\mathbf{z}}_{0}(\mathbf{q})=\left(q_{1} z_{01}, \ldots, q_{n} z_{0 n}\right)$ with $\mathbf{q}=\left(q_{1}, \ldots, q_{n}\right)$. Then we have an inequality:

$$
\left.\frac{d \rho \mathbf{a}(\mathbf{z}(t))}{d t}\right|_{t=1}=\Re\left(\tilde{\mathbf{z}}_{0}(\mathbf{q}), \nabla \rho \mathbf{a}\left(\mathbf{z}_{0}, \overline{\mathbf{z}}_{0}\right)\right)=\Re\left(\tilde{\mathbf{z}}_{0}(\mathbf{q}), 2 \tilde{\mathbf{z}}_{0}(\mathbf{a})\right)>0
$$

On the other hand, the mixed real polynomials $h(\mathbf{z}, \overline{\mathbf{z}}), g((\mathbf{z}, \overline{\mathbf{z}})$ are radially weighted homogeneous under the same weight $\mathbf{q}=\left(q_{1}, \ldots, q_{n}\right)$. This implies $h(\mathbf{z}(t)) \equiv g(\mathbf{z}(t)) \equiv 0$ and we have two equalities:

$$
\begin{aligned}
& \left.\frac{d h(\mathbf{z}(t))}{d t}\right|_{t=1}=\Re\left(\tilde{\mathbf{z}}_{0}(\mathbf{q}), \nabla h\left(\mathbf{z}_{0}, \overline{\mathbf{z}}_{0}\right)\right)=0, \\
& \left.\frac{d g(\mathbf{z}(t))}{d t}\right|_{t=1}=\Re\left(\tilde{\mathbf{z}}_{0}(\mathbf{q}), \nabla g\left(\mathbf{z}_{0}, \overline{\mathbf{z}}_{0}\right)\right)=0 .
\end{aligned}
$$

Now we have a contradiction to ( 7) by ([6):

$$
\begin{aligned}
& 0<\Re\left(\tilde{\mathbf{z}}_{0}(\mathbf{q}), \nabla \rho \mathbf{a}\left(\mathbf{z}_{0}, \overline{\mathbf{z}}_{0}\right)\right)= \\
& \alpha \Re\left(\tilde{\mathbf{z}}_{0}(\mathbf{q}), \nabla h\left(\mathbf{z}_{0}, \overline{\mathbf{z}}_{0}\right)\right)+\beta \Re\left(\tilde{\mathbf{z}}_{0}(\mathbf{q}), \nabla g\left(\mathbf{z}_{0}, \overline{\mathbf{z}}_{0}\right)\right)=0 .
\end{aligned}
$$

2.3. Mixed functions of strongly polar weighted homogeneous face type. Consider a mixed function $f(\mathbf{z}, \overline{\mathbf{z}})=\sum_{\nu, \mu} c_{\nu \mu} \mathbf{z}^{\nu} \overline{\mathbf{z}}^{\mu}$. Recall that for a weight vector $P=\left(p_{1}, \ldots, p_{n}\right)$, the face function $f_{P}$ is defined by the linear sum of the monomials with the radial degree is the minimum ([9]). Thus $f_{P}(\mathbf{z}, \overline{\mathbf{z}})$ is a radially weighted homogeneous polynomial with the weight $P$.

2.3.1. Definition. $f$ is called a mixed function of polar positive weighted homogeneous face type if for any weight vector $P$ with $\operatorname{dim} \Delta(P)=n-1$, the face function $f_{P}(\mathbf{z}, \overline{\mathbf{z}})$ is a polar weighted homogeneous polynomial with some weight vector $P^{\prime}\left(P^{\prime}\right.$ need not be $\left.P\right)$ and $\operatorname{pdeg}_{P^{\prime}} f_{P}>0$.

$f$ is called a mixed function of strongly polar positive weighted homogeneous face type if the face function $f_{P}(\mathbf{z}, \overline{\mathbf{z}})$ is a strongly polar positive 
weighted homogeneous polynomial with the same weight vector $P$, for any $P$ with $\operatorname{dim} \Delta(P)=n-1$.

Proposition 5. (1) Assume that $f(\mathbf{z}, \overline{\mathbf{z}})$ is a convenient mixed function of polar positive weighted homogeneous face type. Then for any weight vector $P, f_{P}$ is also polar weighted homogeneous polynomial.

(2) Assume that $f(\mathbf{z}, \overline{\mathbf{z}})$ is a convenient mixed function of strongly polar positive weighted homogeneous face type. Then for any weight vector $P, f_{P}$ is also a strongly polar positive weighted homogeneous polynomial.

Proof. The assertion (1) is obvious, as any face $\Delta$ of $\Gamma(f)$ is a subface of a face of dimension $n-1$. We consider the assertion (2). The assertion is proved by the descending induction on $\operatorname{dim} \Delta(P)$. The assertion for the case $\operatorname{dim} \Delta(P)=n-1$ is the definition itself. Suppose that $\operatorname{dim} \Delta(P)=k$ and the assertion is true for faces with $\operatorname{dim} \Delta \geq k+1$. In the dual Newton diagram, $P$ is contained in the interior of a cell $\Xi$ whose vertices $Q$ satisfies $\operatorname{dim} \Delta(Q) \geq k+1$. This implies $P$ is a linear combination $\sum_{j=1}^{s} a_{j} Q_{j}$ with $a_{j} \geq 0$ and $\operatorname{dim} \Delta\left(Q_{j}\right) \geq k+1$ where $Q_{1}, \ldots, Q_{s}$ are vertices of $\Xi$. This implies also that $\Delta(P)=\cap_{j}^{s} \Delta\left(Q_{j}\right)$. Write $f_{P}(\mathbf{z}, \overline{\mathbf{z}})=\sum_{k} c_{k} \mathbf{z}^{\nu_{k}} \overline{\mathbf{z}}^{\mu_{k}}$. As $f_{Q_{j}}$ is a strongly polar weighted homogeneous polynomial with weight $Q_{j}$,

$$
\operatorname{pdeg}_{Q_{j}} \mathbf{z}^{\nu_{k}} \overline{\mathbf{z}}^{\mu_{k}}=m_{j}, \quad j=1, \ldots, s
$$

where $m_{j}$ is independent of $k$. This implies $f_{P}$ is polar weighted homogeneous polynomial of weight $P$ with polar degree $\sum_{j=1}^{s} a_{j} m_{j}>0$.

As an obvious but important example, we have

Proposition 6. A holomorphic function $f(\mathbf{z}, \overline{\mathbf{z}})$ is a mixed function of strongly polar positive weighted homogeneous face type.

A mixed function of strongly polar weighted homogeneous face type behaves like a non-degenerate holomorphic function. In [7, we have proved a Varchenko type formula for the zeta function.

2.4. Mixed cyclic covering. Consider two non-negative integer vectors $\mathbf{a}=\left(a_{1}, \ldots, a_{n}\right)$ and $\mathbf{b}=\left(b_{1}, \ldots, b_{n}\right)$. We say $\mathbf{a}$ is strictly bigger than $\mathbf{b}$ if $a_{j}>b_{j} \geq 0$ for any $j=1, \ldots, n$. If this is the case, we denote it as $\mathbf{a} \gg \mathbf{b}$. For given $\mathbf{a}, \mathbf{b}$ with $\mathbf{a} \gg \mathbf{b}$, we consider real analytic mapping $\varphi_{\mathbf{a}, \mathbf{b}}$ :

$$
\varphi_{\mathbf{a}, \mathbf{b}}: \mathbb{C}^{n} \rightarrow \mathbb{C}^{n}, \quad \varphi_{\mathbf{a}, \mathbf{b}}(\mathbf{w})=\left(w_{1}^{a_{1}} \bar{w}_{1}^{b_{1}}, \ldots, w_{n}^{a_{n}} \bar{w}_{n}^{b_{n}}\right) .
$$

We call $\varphi_{\mathbf{a}, \mathbf{b}}$ a mixed cyclic covering mapping associated with integer vectors $\mathbf{a}=\left(a_{1}, \ldots, a_{n}\right)$ and $\mathbf{b}=\left(b_{1}, \ldots, b_{n}\right)$. In fact, over $\mathbb{C}^{* n}, \varphi \mathbf{a}, \mathbf{b}: \mathbb{C}^{* n} \rightarrow$ $\mathbb{C}^{* n}$ is a $\prod_{j=1}^{n}\left(a_{j}-b_{j}\right)$-fold polycyclic covering.

We say that $\varphi_{\mathbf{a}, \mathbf{b}}$ is homogeneous if $\mathbf{a}=(a, \ldots, a)$ and $\mathbf{b}=(b, \ldots, b)$ where $a, b$ are integers such that $a>b \geq 0$. In this case, we denote $\varphi_{a, b}$ 
instead of $\varphi_{\mathbf{a}, \mathbf{b}}$ and we call $\varphi_{a, b}$ a homogeneous mixed covering. For a given mixed function $f(\mathbf{z}, \overline{\mathbf{z}})$, the pull-back $g=\varphi_{\mathbf{a}, \mathbf{b}}^{*}(f)$ is defined by

$$
g(\mathbf{w}, \overline{\mathbf{w}})=f \circ \varphi_{\mathbf{a}, \mathbf{b}}(\mathbf{w}, \overline{\mathbf{w}})=f\left(w_{1}^{a_{1}} \bar{w}_{1}^{b_{1}}, \ldots, w_{n}^{a_{n}} \bar{w}_{n}^{b_{n}}\right) .
$$

Proposition 7. Let $f(\mathbf{z}, \overline{\mathbf{z}})$ be a non-degenerate convenient mixed function of polar weighted homogeneous face type. Let $\varphi=\varphi_{\mathbf{a}, \mathbf{b}}$ be the mixed cyclic covering associated with $\mathbf{a}=\left(a_{1}, \ldots, a_{n}\right)$ and $\mathbf{b}=\left(b_{1}, \ldots, b_{n}\right)$ as above. Consider the pull-back $g(\mathbf{w}, \overline{\mathbf{w}})=f(\varphi(\mathbf{w}, \overline{\mathbf{w}}))$. Then $g(\mathbf{w}, \overline{\mathbf{w}})$ is a convenient non-degenerate mixed function of polar weighted homogeneous face type.

If $f$ is of strongly polar positive weighted homogeneous face type and $\varphi=$ $\varphi_{a, b}$ is a homogeneous mixed covering mapping, $g$ is also of strongly polar positive weighted homogeneous face type.

Proof. Let $P$ be a weight vector and consider $f_{P}(\mathbf{z}, \overline{\mathbf{z}})$. It is a radially weighted homogeneous polynomial under the weight $P$. Let $R=\left(r_{1}, \ldots, r_{n}\right)$ be the polar weight of $f_{P}$. Let $d_{r}$ and $d_{p}$ be the radial and polar degree of $f_{P}$. We consider the normalized weight $Q=\left(q_{1}, \ldots, q_{n}\right) \in \mathbb{Q}^{n}$ and $S=\left(s_{1}, \ldots, s_{n}\right) \in \mathbb{Q}^{n}$ where $q_{j}=p_{j} / d_{r}$ and $s_{j}=r_{j} / d_{p}$. We consider also the normalized weights $\hat{Q}=\left(\hat{q}_{1}, \ldots, \hat{q}_{n}\right)$ and $\hat{S}=\left(\hat{s}_{1}, \ldots, \hat{s}_{n}\right)$ where

$$
\hat{q}_{j}=q_{j} /\left(a_{j}+b_{j}\right), \hat{s}_{j}=s_{j} /\left(a_{j}-b_{j}\right), \quad j=1, \ldots, n .
$$

Consider a monomial $M=z_{1}^{m_{1}} \bar{z}_{1}^{\ell_{1}} \ldots z_{n}^{m_{n}} \bar{z}_{n}^{\ell_{n}}$ in $f_{P}$, i.e. $\operatorname{deg}_{Q} M=1, \operatorname{pdeg}_{S} M=$ 1. Consider the pull-back of $M$,

$$
M^{\prime}=\varphi^{*} M=\prod_{j=1}^{n}\left(w_{j}^{a_{j}} \bar{w}_{j}^{b_{j}}\right)^{m_{j}}\left(\bar{w}_{j}^{a_{j}} w_{j}^{b_{j}}\right)^{\ell_{j}}
$$

Then by an easy calculation, we have

$$
\begin{aligned}
\operatorname{deg}_{\hat{Q}} M^{\prime} & =\sum_{j=1}^{n} q_{j}\left(m_{j}+\ell_{j}\right)=\operatorname{deg}_{Q} M=1 \\
\operatorname{pdeg}_{\hat{S}} M^{\prime}= & \sum_{j=1}^{n} s_{j}\left(m_{j}-\ell_{j}\right)=\operatorname{pdeg}_{S} M=1
\end{aligned}
$$

This implies that $\varphi^{*} f_{P}=g_{\hat{Q}}$ is a radially weighted homogeneous polynomial by the normalized weight vector $\hat{Q}$ and $\varphi^{*} f_{P}$ is a polar weighted homogeneous polynomial by the normal weight vector $\hat{S}$. Non-degeneracy is the result of the commutative diagram:

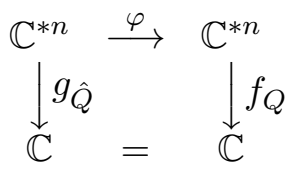

We observe that if $\varphi=\varphi_{a, b}$ and $p_{j}=r_{j}$,

$$
\hat{s}_{j}(a-b) d_{p}=r_{j}=p_{j}=\hat{q}_{j}(a+b) d_{r} .
$$


which implies that $\varphi^{*} f_{P}$ is strongly polar weighted homogeneous.

As holomorphic functions are obviously mixed functions of strongly polar weighted homogeneous face type, we have:

Corollary 8. Assume that $f(\mathbf{z})$ is a convenient non-degenerate holomorphic function and $g(\mathbf{w}, \overline{\mathbf{w}})=\varphi^{*} f(\mathbf{w}, \overline{\mathbf{w}})$ with $\varphi=\varphi_{\mathbf{a}, \mathbf{b}}$. Then $g(\mathbf{w}, \overline{\mathbf{w}})$ is a convenient non-degenerate mixed function of polar weighted homogeneous face type. If further $\varphi=\varphi_{a, b}$, homogeneous with $a>b \geq 0, g$ is of strongly polar positive weighted homogeneous face type.

\section{Contact structure}

3.1. Contact structure and a contact submanifold of a sphere. Let $M$ be a smooth oriented manifold of dimension $2 n-1$. A contact structure on $M$ is a hyperplane distribution $\xi$ in the tangent bundle $T M(M \ni p \mapsto$ $\left.\xi(p) \subset T_{p} M\right)$ which is induced by a global 1 -form $\alpha$ by $\xi(p)=\operatorname{Ker} \alpha$ such that $\alpha \wedge(d \alpha)^{n-1}$ is nowhere vanishing $(2 n-1)$ form. We say $\alpha$ is positive if $\alpha \wedge(d \alpha)^{n-1}$ is a positive form.

We consider the radius function $\rho(\mathbf{z}, \overline{\mathbf{z}})=z_{1} \bar{z}_{1}+\cdots+z_{n} \bar{z}_{n}$. The level manifold $\rho^{-1}\left(r^{2}\right)$ is nothing but the sphere $\mathbb{S}_{r}$. On $\mathbb{S}_{r}$, we consider the canonical contact structure $\xi$ defined by the contact form $\alpha:=-d^{c} \rho=$ $-d \rho \circ J$ where $J$ is the complex structure. More explicitly,

$$
\alpha=\sum_{j=1}^{n}-i\left(\bar{z}_{j} d z_{j}-z_{j} d \bar{z}_{j}\right)=2 \sum_{j=1}^{n}\left(x_{j} d y_{j}-y_{j} d x_{j}\right) .
$$

$\xi(\mathbf{z})$ is nothing but the complex hyperplane which is hermitian orthogonal to $\mathbf{z}: \xi(\mathbf{z})=\{\mathbf{v} \mid(\mathbf{v}, \mathbf{z})=0\}$.

Let $\omega=d \alpha=-d d^{c} \varphi$. Then $\omega$ is explicitly written as

$$
\omega(\mathbf{z})=2 i \sum_{j=1}^{n} d z_{j} \wedge \bar{d} z_{j}=4 \sum_{j=1}^{n} d x_{j} \wedge d y_{j}
$$

and $\omega$ defines a symplectic structure on $\xi$. We have a canonical equality $([2]):$

$$
4 \Re(\mathbf{u}, \mathbf{v})=\omega(\mathbf{u}, J \mathbf{v}), \quad \mathbf{u}, \mathbf{v} \in T \mathbb{S}_{r} .
$$

The Reeb vector field $R \in \Gamma\left(\mathbb{S}_{r}, T \mathbb{S}_{r}\right)$ is defined by the property:

$$
\alpha(R)=1, \quad \iota_{R}(\omega)=0 .
$$

Here $\iota_{R}$ is the inner derivative by $R$. In our case,

$$
\begin{aligned}
R(\mathbf{z}) & =\frac{i \mathbf{z}}{2 \rho(\mathbf{z})}, \quad \text { or as a tangent vector } \\
& =\frac{i}{2 \rho(\mathbf{z})} \sum_{j=1}^{n}\left(z_{j} \frac{\partial}{\partial z_{j}}-\bar{z}_{j} \frac{\partial}{\partial \bar{z}_{j}}\right)=\frac{1}{2 r^{2}} \sum_{j=1}^{n}\left(x_{j} \frac{\partial}{\partial y_{j}}-y_{j} \frac{\partial}{\partial x_{j}}\right) .
\end{aligned}
$$


We consider a real codimension two submanifold $K \subset \mathbb{S}_{r}$. We say $K$ is a (positive) contact submanifold of $\mathbb{S}_{r}$ if the restriction $\alpha_{\mid K}$ defines a contact submanifold, i.e. $(2 n-3)$-form $\alpha \wedge(d \alpha)^{n-2}$ is nowhere vanishing form (respectively positive form) of $K$.

3.2. Remarks on the orientation. The orientation of $\mathbb{S}_{r}$ is given as follows. A $(2 n-1)$-form $\Omega$ is positive if and only if $d \rho \wedge \Omega$ is a positive form of $\mathbb{C}^{n}$. Thus $\alpha \wedge(d \alpha)^{n-1}$ is positive. Let $f(\mathbf{z}, \overline{\mathbf{z}})=g(\mathbf{z}, \overline{\mathbf{z}})+i h(\mathbf{z}, \overline{\mathbf{z}})$ be a non-degenerate mixed function with an isolated mixed singularity at the origin. Let $K_{r}=f^{-1}(0) \cap \mathbb{S}_{r}$ with a sufficiently small $r$. The orientation of $K_{r}$ is given by an $(2 n-3)$ form $\Omega^{\prime}$ such that $d \rho \wedge \Omega^{\prime} \wedge d g \wedge d h$ is a positive form of $\mathbb{C}^{n}$.

3.3. Contact structure on mixed links. First we prepare a lemma. Put $f(\mathbf{z}, \overline{\mathbf{z}})=g(\mathbf{z}, \overline{\mathbf{z}})+i h(\mathbf{z}, \overline{\mathbf{z}})$, where $g, h$ are real valued mixed functions. We use hereafter the following notation for simplicity .

$$
f_{z_{j}}=\frac{\partial f}{\partial z_{j}}, \quad f_{\bar{z}_{j}}=\frac{\partial f}{\partial \bar{z}_{j}} .
$$

Lemma 9. (1) $d \rho \wedge \alpha$ is given as follows.

$$
d \rho \wedge \alpha=i \sum_{a, b=1}^{n} A_{a, \bar{b}} d z_{a} \wedge d \bar{z}_{b}, \quad A_{a, \bar{b}}=2 \bar{z}_{a} z_{b} .
$$

(2) The two form $d g \wedge d h$ can be written as follows.

$$
d g \wedge d h=i \sum_{a, b=1}^{n} B_{a, \bar{b}} d z_{a} \wedge d \bar{z}_{b}+R
$$

where

$$
B_{a, \bar{b}}=\frac{1}{2}\left(f_{z_{a}} \overline{f_{z_{b}}}-\overline{f_{\bar{z}_{a}}} f_{\bar{z}_{b}}\right)
$$

$R$ is a linear combination of two forms $d z_{a} \wedge d z_{b}$ and $d \bar{z}_{a} \wedge d \bar{z}_{b}$.

Proof. The assertion (1) is a result of a simple calculation:

$$
d \rho \wedge \alpha=\left(\sum_{j=1}^{n}\left(z_{j} d \bar{z}_{j}+\bar{z}_{j} d z_{j}\right)\right) \wedge\left(i \sum_{k=1}^{n} z_{k} d \bar{z}_{k}-\bar{z}_{k} d z_{k}\right) .
$$

For (2), we use the equality

$$
g=\frac{1}{2}(f+\bar{f}), \quad h=\frac{-i}{2}(f-\bar{f}) .
$$

Thus we have

$$
\begin{array}{r}
d g=\frac{1}{2} \sum_{j=1}^{n}\left\{\left(f_{z_{j}}+\bar{f}_{z_{j}}\right) d z_{j}+\left(f_{\bar{z}_{j}}+\bar{f}_{\bar{z}_{j}}\right) d \bar{z}_{j}\right\} \\
\left.d h=\frac{-i}{2} \sum_{j=1}^{n}\left\{\left(f_{z_{j}}-\bar{f}_{z_{j}}\right) d z_{j}+\left(f_{\bar{z}_{j}}-\bar{f}_{\bar{z}_{j}}\right) d \bar{z}_{j}\right)\right\}
\end{array}
$$


As $\bar{f}_{\bar{z}_{j}}=\overline{f_{z_{j}}}$ and $\bar{f}_{z_{j}}=\overline{f_{\bar{z}_{j}}}$, the assertion follows by a simple calculation.

Corollary 10. The four form $d \rho \wedge \alpha \wedge d g \wedge d h$ is given as follows.

$$
\begin{gathered}
d \rho \wedge \alpha \wedge d g \wedge d h=-\sum_{a, b=1}^{n} C_{a, b} d z_{a} \wedge d \bar{z}_{a} \wedge d z_{b} \wedge d \bar{z}_{b}+S \\
C_{a, b}=\left|\bar{z}_{a} f_{z_{b}}-\bar{z}_{b} f_{z_{a}}\right|^{2}-\left|z_{a} f_{\bar{z}_{b}}-z_{b} f_{\bar{z}_{a}}\right|^{2}
\end{gathered}
$$

where $S$ is a linear combination of other type of four forms.

Proof. Write

$$
d \rho \wedge \alpha \wedge d g \wedge d h=-\sum_{a, b=1}^{n} C_{a, b} d z_{a} \wedge d \bar{z}_{a} \wedge d z_{b} \wedge d \bar{z}_{b}+S .
$$

Then by Lemma 9, we have

$$
\begin{aligned}
C_{a, b}= & A_{a, \bar{a}} B_{b, \bar{b}}+A_{b, \bar{b}} B_{a, \bar{a}}-A_{a, \bar{b}} B_{b, \bar{a}}-A_{b, \bar{a}} B_{a, \bar{b}} \\
= & \left|z_{a}\right|^{2}\left(\left|f_{z_{b}}\right|^{2}-\left|f_{\bar{b}}\right|^{2}\right)+\left|z_{b}\right|^{2}\left(\left|f_{z_{a}}\right|^{2}-\left|f_{\bar{z}_{a}}\right|^{2}\right) \\
& \quad-2 \bar{z}_{a} z_{b}\left(f_{z_{b}} \overline{f_{z_{a}}}-\overline{f_{\bar{z}_{b}}} f_{\bar{a}}\right)-2 \bar{z}_{b} z_{a}\left(f_{z_{a}} \overline{f_{z_{b}}}-\overline{f_{\bar{z}_{a}}} f_{\bar{b}}\right) \\
= & \left(z_{a} \overline{f_{b}}-z_{b} \overline{f_{a}}\right)\left(\bar{z}_{a} f_{b}-\bar{z}_{b} f_{a}\right)-\left(z_{b} \overline{f_{a}}-z_{a} \overline{f_{b}}\right)\left(\bar{z}_{b} f_{a}-\bar{z}_{a} f_{b}\right) \\
= & \left|\bar{z}_{a} f_{z_{b}}-\bar{z}_{b} f_{z_{a}}\right|^{2}-\left|z_{a} f_{\bar{z}_{b}}-z_{b} f_{\bar{z}_{a}}\right|^{2} .
\end{aligned}
$$

Define $C(\mathbf{z}, \overline{\mathbf{z}}):=\sum_{1 \leq a<b \leq n} C_{a, b}(\mathbf{z}, \overline{\mathbf{z}})$. By Corollary 10 and an easy computation gives the following.

Corollary 11. We have

$$
\begin{aligned}
& d \rho \wedge \alpha \wedge d \alpha^{n-2} \wedge d g \wedge d h(\mathbf{z}, \overline{\mathbf{z}}) \\
= & i^{n} 2^{n-2}(n-2) ! C(\mathbf{z}, \overline{\mathbf{z}}) d z_{1} \wedge \bar{z}_{1} \wedge \cdots \wedge d z_{n} \wedge d \bar{z}_{n} \\
= & 4^{n-1}(n-2) ! C(\mathbf{z}, \overline{\mathbf{z}}) d x_{1} \wedge d y_{1} \wedge \cdots \wedge d x_{n} \wedge d y_{n} .
\end{aligned}
$$

3.4. Holomorphic-like mixed function. Let $U$ be an open neighborhood of the origin. A mixed function $f(\mathbf{z}, \overline{\mathbf{z}})$ which is defined on $U$ with an isolated mixed singularity at the origin is called holomorphic-like (respectively antiholomorphic-like) if for any $\mathbf{z} \in f^{-1}(0) \cap U$,

$$
\begin{aligned}
C(\mathbf{z}, \overline{\mathbf{z}}) & =\sum_{1 \leq a<b \leq n} C_{a, b} \geq 0 \\
C_{a, b} & =\left|z_{a} \overline{f_{z_{b}}}-z_{b} \overline{f_{z_{a}}}\right|^{2}-\left|z_{a} f_{\bar{z}_{b}}-z_{b} f_{\bar{z}_{a}}\right|^{2}
\end{aligned}
$$

( or respectively $C(\mathbf{z}, \overline{\mathbf{z}})=\sum_{1 \leq a<b \leq n} C_{a, b} \leq 0$ ).

We say that $f(\mathbf{z}, \overline{\mathbf{z}})$ is strictly holomorphic-like (resp. strictly anti-holomorphiclike ) in $U$ if $C(\mathbf{z}, \overline{\mathbf{z}})>0$ (resp. $C(\mathbf{z}, \overline{\mathbf{z}})<0$ ) on any smooth point $\mathbf{z} \in$ $U \cap f^{-1}(0) \backslash\{\mathbf{0}\}$.

Remark. If $f$ is a holomorphic function, $f_{\bar{z}_{j}}=0$ and $C_{a, b} \geq 0$ for any $1 \leq a<b \leq n$. Thus $f(\mathbf{z})$ is obviously (strictly) holomorphic-like. If $f(\overline{\mathbf{z}})$ is an anti-holomorphic function, $f_{z_{j}}=0$ and $f(\mathbf{z})$ is anti-holomorphic-like. 
Lemma 12. Assume that $f(\mathbf{z})$ is a holomorphic function and let $g(\mathbf{w}, \overline{\mathbf{w}})=$ $\varphi^{*} f(\mathbf{w}, \overline{\mathbf{w}})=f(\varphi(\mathbf{w}, \overline{\mathbf{w}}))$ where $\varphi$ is a mixed homogeneous cyclic covering associated with integers $a>b \geq 0$ (respectively $0 \leq a<b$ ). Then $g$ is a holomorphic-like (resp. anti-holomorphic-like) mixed function in a neighborhood of the origin.

Proof. By an easy calculation, we get

$$
\begin{gathered}
g_{w_{j}}=f_{z_{j}}(\varphi(\mathbf{w})) a w_{j}^{a-1} \bar{w}_{j}^{b}, \\
g_{\bar{w}_{j}}=f_{z_{j}}(\varphi(\mathbf{w})) b w_{j}^{a} \bar{w}_{j}^{b-1}
\end{gathered}
$$

and for the case $b \geq 1$ we get

$$
\begin{aligned}
C_{j, k}=\mid a \bar{w}_{j} w_{k}^{a-1} & \bar{w}_{k}^{b} \varphi^{*} f_{z_{k}}-\left.a \bar{w}_{k} w_{j}^{a-1} \bar{w}_{j}^{b} \varphi^{*} f_{z_{j}}\right|^{2} \\
& -\left|b w_{j} w_{k}^{a} \bar{w}_{k}^{b-1} \varphi^{*} f_{z_{k}}-b w_{k} w_{j}^{a} \bar{w}_{j}^{b-1} \varphi^{*} f_{z_{j}}\right|^{2} \\
& =\left(a^{2}-b^{2}\right)\left|w_{j} w_{k}\right|^{2}\left(w_{k}^{a-1} \bar{w}_{k}^{b-1} \varphi^{*} f_{z_{k}}-w_{j}^{a-1} \bar{w}_{j}^{b-1} \varphi^{*} f_{z_{j}}\right)^{2}
\end{aligned}
$$

If $b=0, g$ is a holomorphic function and

$$
C_{j, k}=a^{2}\left|\bar{w}_{j} w_{k}^{a-1} \varphi^{*} f_{z_{k}}-\bar{w}_{k} w_{j}^{a-1} \varphi^{*} f_{z_{j}}\right|^{2} \geq 0 .
$$

We are ready to state the first main theorem.

Theorem 13. Assume that $f(\mathbf{z})$ is a convenient non-degenerate holomorphic function. Consider a mixed homogeneous covering $\varphi=\varphi_{a, b}: \mathbb{C}^{n} \rightarrow \mathbb{C}^{n}$, $\varphi(\mathbf{w}, \overline{\mathbf{w}})=\left(w_{1}^{a} \bar{w}_{1}^{b}, \ldots, w_{n}^{a} \bar{w}_{n}^{b}\right)$ and let $g(\mathbf{w}, \overline{\mathbf{w}})=f(\varphi(\mathbf{w}, \overline{\mathbf{w}}))$. Assume that $a>b>0$ (respectively $0<a<b$ ) and consider the link $K_{r}:=g^{-1}(0) \cap \mathbb{S}_{r}$. Then there exists a positive number $r_{0}$ so that $g$ is strictly holomorphiclike (resp. anti-holomorphic-like) on $B_{r_{0}}$ and $K_{r} \subset \mathbb{S}_{r}$ is a positive (resp. negative) contact submanifold for any $r, 0<r \leq r_{0}$.

If $f(\mathbf{z})$ is weighted homogeneous, $g(\mathbf{w}, \overline{\mathbf{w}})$ is strictly holomorphic-like (resp. anti-holomorphic-like) on $\mathbb{C}^{n}$ and $K_{r} \subset \mathbb{S}_{r}$ is a positive contact submanifold for any $r>0$.

Proof. By the convenience assumption of $f(\mathbf{z}), g(\mathbf{w}, \overline{\mathbf{w}})$ is convenient. As $f(\mathbf{z})$ has an isolated singularity at the origin and the restriction $\varphi: \mathbb{C}^{* I} \rightarrow$ $\mathbb{C}^{* I}$ is a covering mapping for any $I \subset\{1, \ldots, n\}, g^{I}$ has an isolated mixed singularity at the origin. Put $g(\mathbf{w}, \overline{\mathbf{w}})=\Re g(\mathbf{w}, \overline{\mathbf{w}})+i \Im g(\mathbf{w}, \overline{\mathbf{w}})$. As a submanifold of $\mathbb{C}^{n}, K$ is a complete intersection variety defined by three real valued functions $\rho=\Re g=\Im g=0$. As the proof is completely the same, we assume that $a>b>0$. To prove $\alpha \wedge d \alpha^{n-2}$ is positive non-vanishing on $K_{r}$, we can equivalently show that $d \rho \wedge \alpha \wedge d \alpha^{n-2} \wedge d \Re g \wedge d \Im g$ is locally nonvanishing on an arbitrary chosen point $\mathbf{z} \in K_{r}$ and positive. This follows from the fact that by the complete intersection property $\rho, \Re g$, $\Im g$ can be a part of real coordinate system of $\mathbb{C}^{n}$ near any point of $K_{r}$. Namely there 
exist real analytic functions $h_{4}, \ldots, h_{2 n}$ such that $\left(\rho, \Re g, \Im g, h_{4}, \ldots, h_{2 n}\right)$ are local coordinates. By Corollary 11 and Corollary, we have

$$
\begin{aligned}
& d \rho \wedge \alpha \wedge d \alpha^{n-2} \wedge d \Re g \wedge d \Im g(\mathbf{w}, \overline{\mathbf{w}})= \\
& i^{n} 2^{n-2}(n-2) ! C(\mathbf{w}, \overline{\mathbf{w}}) d w_{1} \wedge d \bar{w}_{1} \wedge \cdots \wedge d w_{n} \wedge d \bar{w}_{n}
\end{aligned}
$$

The proof of the theorem is reduced to the following Lemma.

Lemma 14. (1) A smooth link $K=g^{-1}(0) \cap \mathbb{S}_{r}$ is a contact submanifold of $\mathbb{S}_{r}$ if and only if $C(\mathbf{w}, \overline{\mathbf{w}})>0$ on $K$.

(2) Assume that $g=\varphi^{*} f$ be as in Theorem 13 with $a>b>0$. Then there exists a sufficiently small neighborhood $U$ of the origin so that $C(\mathbf{w}, \overline{\mathbf{w}})>0$ for any $\mathbf{w} \in g^{-1}(0) \cap U \backslash\{\mathbf{0}\}$.

If further $f(\mathbf{z})$ is weighted homogeneous, $U$ can be the whole space $\mathbb{C}^{n}$.

Proof. Recall that

$$
C_{j, k}=\left(a^{2}-b^{2}\right)\left|w_{j} w_{k}\right|^{2}\left(w_{k}^{a-1} \bar{w}_{k}^{b-1} \varphi^{*} f_{z_{k}}-w_{j}^{a-1} \bar{w}_{j}^{b-1} \varphi^{*} f_{z_{j}}\right)^{2}
$$

Suppose that $C(\mathbf{w}, \overline{\mathbf{w}})=0$ for any small neighborhood. Applying the Curve Selection Lemma ([5]), we get a real analytic curve $\mathbf{w}(t), 0 \leq t \leq 1$ such that for any $1 \leq j, k \leq n$,

$$
\left\{\begin{array}{l}
\left.w_{j} w_{k}\left(w_{k}^{a-1} \bar{w}_{k}^{b-1} \varphi^{*} f_{z_{k}}-w_{j}^{a-1} \bar{w}_{j}^{b-1} \varphi^{*} f_{z_{j}}\right)\right|_{\mathbf{w}=\mathbf{w}(t)}=0 \\
g(\mathbf{w}(t), \overline{\mathbf{w}}(t)) \equiv 0, \quad \mathbf{w}(t) \in \mathbb{C}^{n} \backslash\{\mathbf{0}\}, \mathbf{t} \neq \mathbf{0} .
\end{array}\right.
$$

Let $I=\left\{j \mid w_{j}(t) \neq 0\right\}$. Note that $|I| \geq 2$ as each coordinate axis is not included in $g^{-1}(0)$ by the convenience assumption. By the non-degeneracy assumption on $f$, there exists $j \in I$ such that $f_{z_{j}}(\varphi(\mathbf{w}(t), \overline{\mathbf{w}}(t))) \neq 0$. This implies by (11),

for any $k \in I$. Take $k \in I$ and put

$$
w_{k}(t) f_{z_{k}}(\varphi(\mathbf{w}(t), \overline{\mathbf{w}}(t))) \neq 0
$$

$$
\gamma(t)=w_{k}^{a-1} \bar{w}_{k}^{b-1} \varphi^{*} f_{z_{k}} \mid \mathbf{w}=\mathbf{w}(t) .
$$

Then $\gamma(t) \not \equiv 0$ and $\gamma(t)$ does not depend on the choice of $k \in I$. Put $v_{j}(t):=\frac{d w_{j}(t)}{d t}$ and take the differential of (11). As $v_{j}=0$ for $j \notin I$, we get

$$
\begin{aligned}
0 & =\frac{d g(\mathbf{w}(t), \overline{\mathbf{w}}(t))}{d t} \\
& =\sum_{j=1}^{n} f_{z_{j}}(\varphi(\mathbf{w}(t), \overline{\mathbf{w}}(t)))\left(a w_{j}(t)^{a-1} \bar{w}_{j}(t)^{b} v_{j}(t)+b w_{j}(t)^{a} \bar{w}_{j}(t)^{b-1} \bar{v}_{j}(t)\right) \\
& =\gamma(t) \sum_{j=1}^{n}\left(a \bar{w}_{j}(t) v_{j}(t)+b w_{j}(t) \bar{v}_{j}(t)\right) \\
& =\gamma(t) \frac{(a+b)}{2} \frac{d\|\mathbf{w}(t)\|^{2}}{d t}
\end{aligned}
$$


Thus $\frac{d\|\mathbf{w}(t)\|^{2}}{d t} \equiv 0$. The last equality is derived from

$$
\frac{d\left(\|\mathbf{w}(t)\|^{2}\right.}{d t}=\sum_{j=1}^{n}\left(w_{j}(t) \bar{v}_{j}(t)+\bar{w}_{j}(t) v_{j}(t)\right)=2 \Re \sum_{j=1}^{n} w_{j}(t) \bar{v}_{j}(t) .
$$

This implies that $\|\mathbf{w}(t)\|$ is constant which is a contradiction to the assumption $\|\mathbf{w}(t)\| \rightarrow 0(t \rightarrow 0)$.

We prove the second assertion. Assume that $f(\mathbf{z})$ is a weighted homogeneous polynomial of degree $d$ with a weight vector $P=\left(p_{1}, \ldots, p_{n}\right)$ and $a>b>1$. Then $g=\varphi^{*} f$ is a strongly polar weighted homogeneous polynomial with $\operatorname{rdeg}_{P} g=(a+b) d$ and $\operatorname{pdeg}_{P} g=(a-b) d$. Put $I=\left\{j \mid w_{j} \neq 0\right\}$. Assume that $C_{j, k}(\mathbf{w}, \overline{\mathbf{w}})=0$ for any $j, k$ for some $\mathbf{w} \in g^{-1}(0) \backslash\{0\}$. Put

$$
\gamma=w_{k}^{a-1} \bar{w}_{k}^{b-1} \varphi^{*} f_{z_{k}}
$$

for any fixed $k \in I$. Note that $\gamma$ is independent of $k \in I$. As $f^{I}$ (and also $g^{I}$ ) has an isolated singularity at the origin, $\gamma \neq 0$. Then this implies that

$$
g_{w_{j}}=a w_{j}^{a-1} \bar{w}_{j}^{b} \varphi^{*} f_{z_{j}}=a \gamma \bar{w}_{j}, j \in I
$$

and by Euler equality (5), we get a contradiction

$$
\begin{aligned}
0=\operatorname{adg}(\mathbf{w}, \overline{\mathbf{w}}) & =\sum_{j=1}^{n} p_{j} w_{j} g_{w_{j}} \\
& =\sum_{j \in I} p_{j} w_{j} g_{w_{j}} \\
& =\gamma a \sum_{j \in I} p_{j}\left|w_{j}\right|^{2} \neq 0 .
\end{aligned}
$$

Corollary 15. Assume that $f(\mathbf{z})$ is a holomorphic function with isolated singularity at the origin. Consider the link $K_{r}:=g^{-1}(0) \cap \mathbb{S}_{r}$. Then there exists a positive number $r_{0}$ so that $K_{r} \subset \mathbb{S}_{r}$ is a positive contact submanifold for any $0<r \leq r_{0}$.

If $f(\mathbf{z})$ is weighted homogeneous, $K_{r} \subset \mathbb{S}_{r}$ is a positive contact submanifold for any $r>0$.

Proof. The proof is parallel to that of Lemma 14. Recall that

$$
C_{j, k}=\left|a \bar{z}_{j} f_{z_{k}}-a \bar{z}_{k} f_{z_{j}}\right|^{2} \text {. }
$$

We do the same argument. If $\left\{\mathbf{z} \in \mathbb{C}^{n} \mid C(\mathbf{z}, \overline{\mathbf{z}})=0\right\} \cap f^{-1}(0)$ is not isolated at the origin, we take an analytic curve $\mathbf{z}(t) \in\{\mathbf{z} \mid C(\mathbf{z}, \overline{\mathbf{z}})=0\} \cap f^{-1}(0)$ as above. Putting $I=\left\{j \mid z_{j}(t) \neq 0\right\}$ as above, we get $|I| \geq 2$. As $f \mid \mathbb{C}^{I}$ has an isolated singularity, we can assume that $f_{z_{k}}(\mathbf{z}(t)) \neq 0$ for some $k \in I$. Take $j \in I$ with $j \neq k$. Then $C_{j, k}=0$ implies that $\bar{z}_{j}(t) f_{z_{k}}(\mathbf{z}(t)) \neq 0$ and thus $k \in I$ and $f_{z_{j}}(\mathbf{z}(t)) \neq 0$. Put $c(\mathbf{z})=f_{z_{k}}(\mathbf{z}) / \bar{z}_{k}$ for a fixed $k \in I$. This is a 
non-zero and independent of $k \in I$. Note that $v_{k}(t)=0$ for $k \notin I$. Therefore we get

$$
\begin{aligned}
0 & =\frac{d f(\mathbf{z}(t))}{d t}=\sum_{j=1}^{n} f_{z_{j}}(\mathbf{z}(t)) v_{j}(t) \\
& =\sum_{j \in I} f_{z_{j}}(\mathbf{z}(t)) v_{j}(t)=c(\mathbf{z}(t)) \sum_{j \in I} \bar{z}_{j}(t) v_{j}(t)
\end{aligned}
$$

and we get the same contradiction $\frac{d\|\mathbf{z}(t)\|^{2}}{d t} \equiv 0$.

Finally assume further $f(\mathbf{z})$ is weighted homogeneous of degree $d$ with weight vector $P=\left(p_{1}, \ldots, p_{n}\right)$. Put $I=\left\{j \mid z_{j} \neq 0\right\}$ as above and put $c(\mathbf{z})=f_{z_{k}}(\mathbf{z}) / \bar{z}_{k}$ for $k \in I$. Then $f_{z_{j}}(\mathbf{z})=c(\mathbf{z}) \bar{z}_{k}$ and we get the same contradiction:

$$
0=f(\mathbf{z})=\sum_{j=1}^{n} p_{j} z_{j} f_{z_{j}}(\mathbf{z})=\sum_{j=1}^{n} c(\mathbf{z}) p_{j}\left|z_{j}\right|^{2} \neq 0 .
$$

Remark 16. Corollary 15 gives a simple proof of holomorphic link to be a contact submanifold without using the strict pseudo-convex property.

\section{Open BoOK STRUCTURE.}

4.1. Open book. An open book with binding $N$ on an oriented manifold $M$ of dimension $2 n-1$ is a couple $(N, \theta)$ where $N$ is a codimension two submanifold with a trivial normal bundle and $\theta: M \backslash N \rightarrow S^{1}$ is a local trivial smooth fibration where $\theta$ coincide with the angular coordinate of the trivial tubular neighborhood $N \times D_{\delta} \subset M$ ([4, [2]). The orientation of $M$ gives a canonical orientation to the fiber $F_{\eta}:=\theta^{-1}(\eta)$ for each $\eta \in S^{1}$. Restricting the fibration on $M \backslash N \times D_{\delta}$, the fiber $F_{\eta}^{\prime}:=F_{\eta} \cap\left(M \backslash N \times D_{\delta}\right)$ is a manifold with boundary $N$. Thus $N$ has also a canonical orientation.

4.2. Contact structure carried by an open book. Assume that we have a contact form $\xi$ defined by a global 1 -form $\alpha$ as before. We say that a contact structure $\xi$ is carried by an open book $(N, \theta)$ if the following are satisfied $([4])$.

(1) The restriction of $\alpha$ to $N$ is a contact form on $N$.

(2) The two-form $d \alpha$ defines a symplectic form of each fiber $F_{\eta}=\theta^{-1}(\eta)$.

(3) The orientation of $N$ induced by $\alpha$ is the same as that of the boundary of $F_{\eta}$.

Recall that the condition (2) is equivalent to $d \theta(R)>0$ where $R$ is the Reeb vector field $([4,3])$. For further detail about a contact structure carried with an open book and symplectic structures, see H. Geiges [3], Giroux [4], R. Berndt [1] and Caubel-Nemethi-Popescu-Pampu [2]. 
4.3. Milnor open book for mixed functions. Let $g(\mathbf{z}, \overline{\mathbf{z}})$ be a convenient strongly non-degenerate mixed function. Let $V=g^{-1}(0)$ and we assume that $V$ has an isolated mixed singularity at the origin. By Theorem 33 ([9]), we have

Theorem 17. For a sufficiently small $r$, the mapping

$$
g /|g|: \quad \mathbb{S}_{r} \backslash K_{r} \rightarrow S^{1}
$$

is a locally trivial fibration.

By the transversality, we have a trivial tubular neighborhood $K_{r} \times D_{\delta}$ such that the following diagram commutes.

$$
\begin{aligned}
& K_{r} \times D_{\delta} \quad \subset \quad K_{r} \times D_{\delta}^{*} \quad \subset \quad \mathbb{S}_{r} \backslash K_{r} \\
& \underset{D_{\delta}^{*}}{\downarrow p} \quad \stackrel{\text { normal }}{\longrightarrow} \quad \underset{S^{1}}{\downarrow}
\end{aligned}
$$

where $D_{\delta}^{*}=\{\eta \in \mathbb{C}|0 \neq \eta,| \eta \mid \leq \delta\}, p$ is the second projection and normal is the normalization map $\eta \mapsto \eta /|\eta| \mid$. The argument $\theta$ is characterized by the equality:

$$
\log g(\mathbf{z}, \overline{\mathbf{z}})=\log |g(\mathbf{z}, \overline{\mathbf{z}})|+i \theta .
$$

From this and the obvious equality $|g(\mathbf{z}, \overline{\mathbf{z}})|^{2}=g(\mathbf{z}, \overline{\mathbf{z}}) \bar{g}(\mathbf{z}, \overline{\mathbf{z}})$, we have

\section{Proposition 18.}

$$
\begin{aligned}
& \nabla \theta=i\left(\frac{\overline{g_{z_{1}}}}{\bar{g}}-\frac{g_{\bar{z}_{1}}}{g}, \ldots, \frac{\overline{g_{z_{n}}}}{\bar{g}}-\frac{g_{\bar{z}_{n}}}{g}\right), \\
& d \theta=-i\left(\frac{\partial g+\bar{\partial} g}{g}-\frac{\partial \bar{g}+\bar{\partial} \bar{g}}{\bar{g}}\right),
\end{aligned}
$$

where $\partial, \bar{\partial}$ are defined for a mixed function $h$ by

$$
d h=\partial h+\bar{\partial} h, \quad \partial h=\sum_{j=1}^{n} h_{z_{j}} d z_{j}, \quad \bar{\partial} h=\sum_{j=1}^{n} h_{\bar{z}_{j}} d \bar{z}_{j} .
$$

4.4. Contact structure carried by a Milnor open book. We consider the existence problem of the contact structure carried by a Milnor open book for polar weighted homogeneous mixed functions. We consider a homogeneous mixed covering lifting $g(\mathbf{w}, \overline{\mathbf{w}})=f\left(w_{1}^{a} \bar{w}_{1}^{b}, \ldots, w_{n}^{a} \bar{w}_{n}^{b}\right)$ with $a>b \geq 0$ where $f(\mathbf{z})$ is a convenient non-degenerate holomorphic function defined in a neighborhood of the origin.

4.4.1. Strongly polar homogeneous case. First we consider the easy case that $f(\mathbf{z})$ is a homogeneous polynomial of degree $d$. Then $g(\mathbf{w}, \overline{\mathbf{w}})$ is a strongly polar homogeneous polynomial with $\operatorname{rdeg} g=d(a+b)$, pdeg $g=d(a-b)$. In this case, we assert:

Theorem 19. Assume that $f(\mathbf{z})$ is a homogeneous polynomial with $\operatorname{pdeg} f=$ $d>0$. The canonical contact form $\alpha$ and $\omega$ is adapted with the Milnor open book $\theta: S_{r}^{2 n-1} \backslash K_{r} \rightarrow S^{1}$ for any $r>0$. 
Proof. Recall that Reeb vector field $R$ and $d \theta$ are given on $\mathbb{S}_{r}$ by

$$
\begin{aligned}
R(\mathbf{z}) & =\frac{i}{2 r^{2}} \sum_{j=1}^{n}\left(z_{j} \frac{\partial}{\partial z_{j}}-\bar{z}_{j} \frac{\partial}{\partial \bar{z}_{j}}\right) \\
d \theta & =-i\left\{\frac{\partial g+\bar{\partial} g}{g}-\frac{\partial \bar{g}+\bar{\partial} \bar{g}}{\bar{g}}\right\}
\end{aligned}
$$

In fact, we use the polar Euler equality:

$$
\sum_{j=1}^{n}\left(z_{j} g_{z_{j}}-\bar{z}_{j} g_{\bar{z}_{j}}\right)=d(a-b) g
$$

and its conjugate:

$$
\sum_{j=1}^{n}\left(\bar{z}_{j} \bar{g}_{\bar{z}_{j}}-z_{j} \bar{g}_{z_{j}}\right)=d(a-b) \bar{g} .
$$

Using these Euler equalities, we get:

$$
\begin{aligned}
d \theta(R) & =\frac{\partial g(R)+\bar{\partial} g(R)}{g}-\frac{\partial \bar{g}(R)+\bar{\partial} \bar{g}(R)}{\bar{g}} \\
& =\sum_{j=1}^{n} \frac{z_{j} g_{z_{j}}-\bar{z}_{j} g_{\bar{z}_{j}}}{g}-\sum_{j=1}^{n} \frac{z_{j} \bar{g}_{z_{j}}-\bar{z}_{j} \bar{g}_{\bar{z}_{j}}}{\bar{g}} \\
& =2 d(a-b)>0 .
\end{aligned}
$$

This shows that for any radius $r>0$, the canonical contact structure on $K_{r} \subset \mathbb{S}_{r}$ is adapted with the Milnor fibration.

\section{General CaSe}

We are interested in the existence of open book structure adapted to the contact structure which is the restriction of $\alpha$ to the link $K_{r} \subset \mathbb{S}_{r}$ where $K_{r}=g^{-1}(0) \cap \mathbb{S}_{r}$. We have shown that there exists a canonical Milnor fibration on $g /|g|: \mathbb{S}_{r} \backslash\left\{K_{r}\right\} \rightarrow S^{1}$ by [9]. However this fibration is not adapted with the symplectic structure given by $d \alpha$. Therefore we will change the contact form $\alpha$ without changing the contact structure $\xi$ so that the new contact form will be carried by the Milnor open book. We follow the proof of Theorem 3.9 in [2] for the holomorphic functions in CaubelNémethi-Popescu-Pampu.

We modify the contact form $\alpha$ by

$$
\alpha_{c}=e^{-c|g|^{2}} \alpha
$$

with a sufficiently large positive real number $c>0$. This does not change the contact structure $\xi=\operatorname{Ker} \alpha$ but the two form $\omega_{c}=d \alpha_{c}$ is changed as

$$
\omega_{c}=d\left(e^{-c|g|^{2}}\right) \wedge \alpha+e^{-c|g|^{2}} d \alpha
$$


and the corresponding symplectic structure changes. Consider the new Reeb vector field $R_{c}$. Put $H=e^{-c|g|^{2}}$. Put also $R_{c}=k\left(R+S_{c}\right)$ with $S_{c}$ is tangent to $\xi$. Then we get $k H=1$. As

$$
d \alpha_{c}=d H \wedge \alpha+H d \alpha
$$

the condition for $R_{c}$ to be the Reeb vector field $\left.\iota_{R_{c}} d \alpha_{c}\right|_{\xi}=0$ gives the condition:

$$
\left.\iota_{S_{c}} \omega\right|_{\xi}=\left.\frac{d H}{H}\right|_{\xi}=-c d|g|^{2} .
$$

Put $\pi: T_{\mathbf{W}} \mathbb{C}^{n} \rightarrow \xi(\mathbf{w})$ be the hermitian orthogonal projection. Namely, $\pi(\mathbf{v})=\mathbf{v}-(\mathbf{v}, \tilde{R}) \tilde{R}$ and $\tilde{R}=R /\|R\|$. Then (15) implies by (8) that

$$
\begin{aligned}
\left.\iota_{S_{c}} \omega\right|_{\xi} & =-\left.c d|g|^{2}\right|_{\xi}=\left.\Re\left(-c \nabla|g|^{2}, \cdot\right)\right|_{\xi} \\
& =\left.\frac{1}{4} \omega\left(i c \nabla|g|^{2}, \cdot\right)\right|_{\xi}=\left.\omega\left(\pi\left(i c \nabla|g|^{2}\right) / 4, \cdot\right)\right|_{\xi} \\
& =\iota_{\pi\left(i c \nabla|g|^{2}\right) /\left.4 \omega\right|_{\xi} .}
\end{aligned}
$$

As $\omega$ is non-degenerate on $\xi$, we get

$$
S_{c}=\pi\left(i c \nabla|g|^{2} / 4\right)
$$

Thus we get

$$
\begin{aligned}
|g|^{2} d \theta\left(R_{c}\right) & =k|g|^{2} d \theta(R)+k \Re\left(|g|^{2} \nabla \theta, S_{c}\right) \\
& =k|g|^{2} d \theta(R)+k \Re\left(\pi\left(|g|^{2} \nabla \theta\right), \pi\left(i c \nabla|g|^{2} / 4\right)\right) .
\end{aligned}
$$

For simplicity, we introduce two vectors

$$
\begin{aligned}
\nabla_{\partial} g & =\left(g_{w_{1}}, \ldots, g_{w_{n}}\right) \\
\nabla \bar{\partial} g & =\left(g_{\bar{w}_{1}}, \ldots, g_{\bar{w}_{n}}\right) .
\end{aligned}
$$

Remark 20. In our previous paper [8], we used the notation $d g$ and $\bar{d} g$ instead of $\nabla_{\partial} g$ and $\nabla_{\bar{\partial}} g$. We changed notations as the previous notations are confusing with 1-forms $\partial g, \bar{\partial} g$. We use dg not for $\partial g$ but $d g=(\partial+\bar{\partial}) g$.

Recall that

$$
\begin{aligned}
\nabla|g|^{2} & =2 g \overline{\nabla_{\partial} g}+2 \bar{g} \nabla \bar{\partial} g \\
|g|^{2} \nabla \theta & =i g \overline{\nabla_{\partial} g}-i \bar{g} \nabla_{\bar{\partial}} g .
\end{aligned}
$$

Thus $2|g|^{2} \nabla \theta$ and $i \nabla|g|^{2}$ are different in the case of mixed functions. This makes a difficulty. (In the holomorphic function case, they are the same up to a scalar multiplication, as $\nabla \bar{\partial} g$ vanishes.)

Put $\pi^{\prime}: \mathbb{C}^{n} \rightarrow \mathbb{C} \cdot R$ be the orthogonal projection to the complex line $\mathbb{C} \cdot R=\mathbb{C} \cdot \mathbf{w}$ generated by $R$ or $\mathbf{w}$. Namely $\pi^{\prime}(\mathbf{v})=(\mathbf{v}, \mathbf{w}) \mathbf{w} /\|\mathbf{w}\|^{2}=$ 
$(\mathbf{v}, R) R /\|R\|^{2}$. Then $\pi(\mathbf{v})=\mathbf{v}-\pi^{\prime}(\mathbf{v})$. Consider the expression:

$$
\begin{gathered}
g \overline{\nabla \partial g}=\mathbf{v}_{11}+\mathbf{v}_{12}, \quad\left\{\begin{array}{l}
\mathbf{v}_{11}=\pi(g \overline{\nabla \partial g}) \\
\mathbf{v}_{12}=\pi^{\prime}(g \overline{\nabla \partial g})
\end{array}\right. \\
\bar{g} \nabla_{\bar{\partial}} g=\mathbf{v}_{21}+\mathbf{v}_{22}, \quad\left\{\begin{array}{l}
\mathbf{v}_{21}=\pi(\bar{g} \nabla \bar{\partial} g) \\
\mathbf{v}_{12}=\pi^{\prime}(\bar{g} \nabla \bar{\partial} g) .
\end{array}\right.
\end{gathered}
$$

Using this expression, we get

$$
\begin{aligned}
\pi\left(i c \nabla|g|^{2} / 4\right) & =\frac{i c}{2}\left(\mathbf{v}_{11}+\mathbf{v}_{21}\right) \\
\pi\left(|g|^{2} \nabla \theta\right) & =i\left(\mathbf{v}_{11}-\mathbf{v}_{21}\right)
\end{aligned}
$$

Thus we get

$$
\begin{aligned}
|g|^{2} d \theta\left(R_{c}\right) & =k|g|^{2} d \theta(R)+k \Re\left(\pi\left(|g|^{2} \nabla \theta\right), \pi\left(i c \nabla|g|^{2} / 2\right)\right) \\
& =k|g|^{2} d \theta(R)+\frac{c k}{2}\left(\left\|v_{11}\right\|^{2}-\left\|v_{21}\right\|^{2}\right)
\end{aligned}
$$

Here we have used the equality: $\Re\left(\mathbf{v}_{21}, \mathbf{v}_{11}\right)=\Re\left(\mathbf{v}_{11}, \mathbf{v}_{21}\right)$. The key assertion is the following.

Lemma 21. We have the inequality: $\left\|v_{11}\right\|^{2}-\left\|v_{21}\right\|^{2} \geq 0$ and the equality takes place if and only if $\overline{\nabla \partial g}(\mathbf{w}, \overline{\mathbf{w}})=\lambda_{1} \mathbf{w}$ and $\nabla_{\bar{\partial}} g(\mathbf{w}, \overline{\mathbf{w}})=\lambda_{2} \mathbf{w}$ for some $\lambda_{1}, \lambda_{2} \in \mathbb{C}$. In this case, we have also $\nabla \theta=\lambda R$ for some $\lambda \in \mathbb{C}$.

Proof. Let $\mathbf{v}_{1}=g \overline{\nabla \partial g}$ and $\mathbf{v}_{2}=\bar{g} \nabla \bar{\partial} g$. As $\left\{\mathbf{v}_{11}, \mathbf{v}_{12}\right\}$ and $\left\{\mathbf{v}_{12}, \mathbf{v}_{22}\right\}$ are hermitian orthogonal, we have

$$
\left\|\mathbf{v}_{11}\right\|^{2}=\left\|\mathbf{v}_{1}\right\|^{2}-\left\|\mathbf{v}_{12}\right\|^{2}, \quad\left\|\mathbf{v}_{21}\right\|^{2}=\left\|\mathbf{v}_{2}\right\|^{2}-\left\|\mathbf{v}_{22}\right\|^{2}
$$

We go now further precise expression. Put

$$
\mathbf{v}_{1}=\left(v_{1}^{1}, \ldots, v_{1}^{n}\right), \quad \mathbf{v}_{2}=\left(v_{2}^{1}, \ldots, v_{2}^{n}\right)
$$

Then we have

$$
\begin{aligned}
v_{1}^{j} & =g(\mathbf{w}, \overline{\mathbf{w}}) \overline{f_{z_{j}}(\varphi(\mathbf{w}, \overline{\mathbf{w}}))} a \bar{w}_{j}^{a-1} w_{j}^{b} \\
v_{2}^{j} & =\bar{g}(\mathbf{w}, \overline{\mathbf{w}}) f_{z_{j}}(\varphi(\mathbf{w}, \overline{\mathbf{w}})) b w_{j}^{a} \bar{w}_{j}^{b-1} .
\end{aligned}
$$


Recall that $R=i \mathbf{w} / 2 \rho(\mathbf{w})$. Thus

$$
\begin{aligned}
& \mathbf{v}_{12}=\left(\sum_{j=1}^{n} g(\mathbf{w}, \overline{\mathbf{w}}) \overline{f_{z_{j}}(\varphi(\mathbf{w}, \overline{\mathbf{w}}))} a \bar{w}_{j}^{a} w_{j}^{b}\right) \mathbf{w} /\|\mathbf{w}\|^{2} \\
& =\operatorname{ag}(\mathbf{w}, \overline{\mathbf{w}})\left(\sum_{j=1}^{n} \overline{f_{z_{j}}}(\varphi(\mathbf{w}, \overline{\mathbf{w}})) \bar{w}_{j}^{a} w_{j}^{b}\right) \mathbf{w} /\|\mathbf{w}\|^{2} \\
& \mathbf{v}_{22}=\left(\sum_{j=1}^{n} \bar{g}(\mathbf{w}, \overline{\mathbf{w}}) f_{z_{j}}(\varphi(\mathbf{w}, \overline{\mathbf{w}})) b w_{j}^{a} \bar{w}_{j}^{b}\right) \mathbf{w} /\|\mathbf{w}\|^{2}
\end{aligned}
$$

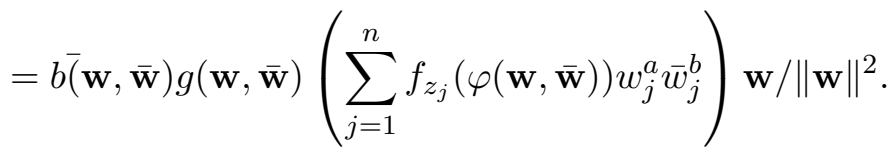

Thus we get

$$
\begin{aligned}
0 \leq\left\|\mathbf{v}_{11}\right\|^{2}= & \left\|\mathbf{v}_{1}\right\|^{2}-\left\|\mathbf{v}_{12}\right\|^{2} \\
= & a^{2}|g|^{2} \sum_{j=1}^{n}\left|f_{z_{j}}\right|^{2}\left|w_{j}\right|^{2(a+b-1)}-a^{2}|g|^{2}\left|\sum_{j=1}^{n} \overline{f_{z_{j}}} \bar{w}_{j}^{a} w_{j}^{b}\right|^{2} \\
= & a^{2}|g|^{2}(\gamma-\beta) \\
0 \leq\left\|\mathbf{v}_{21}\right\|^{2}= & \left\|\mathbf{v}_{2}\right\|^{2}-\left\|\mathbf{v}_{22}\right\|^{2} \\
= & b^{2}|g|^{2} \sum_{j=1}^{n}\left|f_{z_{j}}\right|^{2}\left|w_{j}\right|^{2(a+b-1)}-b^{2}|g|^{2}\left|\sum_{j=1}^{n} f_{z_{j}} w_{j}^{a} \bar{w}_{j}^{b}\right|^{2} \\
= & b^{2}|g|^{2}(\gamma-\beta) \\
& \text { where }\left\{\begin{array}{l}
\gamma=\sum_{j=1}^{n}\left|f_{z_{j}}\right|^{2}\left|w_{j}\right|^{2(a+b-1)} \\
\beta=\left|\sum_{j=1}^{n} \overline{f_{z_{j}}} \bar{w}_{j}^{a} w_{j}^{b}\right|^{2} .
\end{array}\right.
\end{aligned}
$$

Thus $\gamma \geq \beta$ and we have

$$
\left\|\mathbf{v}_{11}\right\|^{2}-\left\|\mathbf{v}_{21}\right\|^{2}=\left(a^{2}-b^{2}\right)|g|^{2}(\gamma-\beta) \geq 0
$$

and the equality holds if and only if $\gamma=\beta$. This is equivalent to $\left\|\mathbf{v}_{11}\right\|=$ $\left\|\mathbf{v}_{21}\right\|=0$ and this implies $\overline{\nabla \partial g}(\mathbf{w}, \overline{\mathbf{w}})=\lambda_{1} \mathbf{w}$ and $\nabla \bar{\partial} g(\mathbf{w}, \overline{\mathbf{w}})=\lambda_{2} \mathbf{w}$ for some $\lambda_{1}, \lambda_{2} \in \mathbb{C}$. The last assertion follows from $|g|^{2} \nabla \theta=i\left(\mathbf{v}_{1}-\mathbf{v}_{2}\right)$.

5.1. Main theorem. Now we are ready to state our main theorem. Let $\varphi(\mathbf{w}, \overline{\mathbf{w}})=\left(w_{1}^{a} \bar{w}_{1}^{b}, \ldots, w_{n}^{a} \bar{w}_{n}^{b}\right)$ with $a>b>0$ as before.

Main Theorem 22. Assume that $f(\mathbf{z})$ is a convenient non-degenerate holomorphic function so that $g(\mathbf{w}, \overline{\mathbf{w}})=\varphi^{*} f(\mathbf{w}, \overline{\mathbf{w}})$ is a convenient nondegenerate mixed function of strongly polar weighted homogeneous face type. 
Then there exists a positive number $r_{0}$ such that the Milnor open book $f /|f|: \mathbb{S}_{r} \backslash K_{r} \rightarrow S^{1}$ carries a contact structure for any $r>0$ with $r \leq r_{0}$.

If further $f(\mathbf{z})$ is a weighted homogeneous polynomial, we can take $r_{0}=\infty$ and any $r>0$.

Proof. For the proof, we do the same discussion as that of Caubel-NémethiPopescu-Pampu [2]. Let

$$
Z_{\delta}:=\left\{\mathbf{w} \in S_{r}^{2 n-1} \backslash V_{\delta} \mid d \theta(R) \leq 0\right\}, \quad V_{\delta}=S_{r}^{2 n-1} \cap g^{-1}\left(D_{\delta}\right)
$$

where $\delta$ is sufficiently small so that $f^{-1}(0)$ and $\mathbb{S}_{r}$ are transverse and $V_{\delta}$ is a trivial tubular neighborhood. Let $\alpha_{c}$ and $R_{c}$ be as before. As we have shown that

$$
|g|^{2} d \theta\left(R_{c}\right)=k|g|^{2} d \theta(R)+\frac{c k}{2}\left(\left\|v_{11}\right\|^{2}-\left\|v_{21}\right\|^{2}\right)
$$

with $k=1 / e^{-c|g|^{2}}$ and the second term is non-negative and the equality holds (i.e. $\left\|v_{11}\right\|^{2}-\left\|v_{21}\right\|^{2}=0$ ) if and only if $\nabla \theta=\lambda R$. In this case, $d \theta(R)=$ $\Re \lambda\|R\|^{2}$ and $d \theta(R)$ is positive if $\Re \lambda>0$. Thus taking sufficiently large $c>0$, we only need to show that $\nabla \theta$ and $R$ are linearly independent on $Z_{\delta}$. Thus the following lemma completes the proof. Compare with Proposition $3.8([2])$.

Lemma 23. Assume that $\nabla \theta(\mathbf{w})=\lambda R(\mathbf{w})$ on $\mathbf{w} \in Z_{\delta}$ for some $\lambda \in \mathbb{C}$.

(1) Assume that $f(\mathbf{z})$ is convenient non-degenerate weighted homogeneous polynomial. Then $\lambda$ is a positive real number.

(2) If $f(\mathbf{z})$ is not weighted homogeneous but a convenient non-degenerate mixed function of strongly polar weighted homogeneous face type, there exists a positive number $r_{0}$ such that $\Re \lambda$ is positive for any $\mathbf{w} \in \mathbb{S}_{r} \backslash g^{-1}(0)$ and $r \leq r_{0}$.

Proof. (1) Assume that $f(\mathbf{z})$ is a weighted homogeneous of degree $d$ with weight vector $P=\left(p_{1}, \ldots, p_{n}\right)$. Let $m_{r}=(a+b) d$ and $m_{p}=(a-b) d$, the radial and polar degree of $g(\mathbf{w}, \overline{\mathbf{w}})$. The assumption says that

$$
\lambda w_{j}=\frac{1}{\bar{g}(\mathbf{w}, \overline{\mathbf{w}})} \overline{g_{w_{j}}}(\mathbf{w}, \overline{\mathbf{w}})-\frac{1}{g(\mathbf{w}, \overline{\mathbf{w}})} g_{\bar{w}_{j}}(\mathbf{w}, \overline{\mathbf{w}})
$$

for $j=1, \ldots, n$. Taking the summation of $(28) \times p_{j} \bar{w}_{j}$ for $j=1, \ldots, n$, we get:

$$
\begin{aligned}
\lambda \sum_{j=1}^{n} p_{j}\left|w_{j}\right|^{2} & =\frac{1}{\bar{g}(\mathbf{w}, \overline{\mathbf{w}})} \sum_{j=1}^{n} p_{j} \bar{w}_{j} \overline{g_{w_{j}}}(\mathbf{w}, \overline{\mathbf{w}})-\frac{1}{g(\mathbf{w}, \overline{\mathbf{w}})} \sum_{j=1}^{n} p_{j} \bar{w}_{j} g_{\bar{w}_{j}}(\mathbf{w}, \overline{\mathbf{w}}) \\
& =\left(m_{r}+m_{p}\right)-\left(m_{r}-m_{p}\right)=2 m_{p}>0
\end{aligned}
$$

by the strong Euler equalities (5). This implies $\lambda$ is a positive number.

(2) General case. Assume that $g(\mathbf{w}, \overline{\mathbf{w}})$ is a convenient non-degenerate mixed function of strongly polar weighted homogeneous face type. Assume that the assertion (2) does not hold. Using Curve Selection Lemma ([6, 5]), 
we can find a real analytic curve $\mathbf{w}(t) \in \mathbb{C}^{n} \backslash f^{-1}(0)$ for $0<t \leq \varepsilon$ and Laurent series $\lambda(t)$ such that

$$
\nabla \theta(\mathbf{w}(t))=\lambda(t) R(\mathbf{w}(t))
$$

such that $\nabla \theta(R(\lambda(t))) \leq 0$. We show this give a contradiction by showing $\lim _{t \rightarrow 0} \arg \lambda(t)=0$.

Let $I=\left\{j \mid w_{j}(t) \not \equiv 0\right\}$. Then $|I| \geq 2$ and we restrict our the discussion to the coordinate subspace $\mathbb{C}^{I}$ and $g^{I}=\left.g\right|_{\mathbb{C}^{I}}$. For the notation's simplicity, we assume that $I=\{1, \ldots, n\}$ hereafter. Consider the Taylor (Laurent) expansions:

$$
\begin{array}{r}
w_{j}(t)=a_{j} t^{p_{j}}+(\text { higher terms }), \quad j=1, \ldots, n, \\
\lambda(t)=\lambda_{0} t^{\ell}+(\text { higher terms }), \\
g(\mathbf{w}(t), \overline{\mathbf{w}}(t))=g_{0} t^{d}+(\text { higher terms })
\end{array}
$$

where $a_{j}, \lambda_{0}, g_{0} \neq 0$ and $p_{j} \in \mathbb{N}, \ell \in \mathbb{Z}$. Then the equality (29) says

$$
\lambda(t) w_{j}(t)=\frac{\overline{g_{w_{j}}}(\mathbf{w}(t), \overline{\mathbf{w}}(t))}{\bar{g}(\mathbf{w}(t), \overline{\mathbf{w}}(t))}-\frac{g_{\bar{w}_{j}}(\mathbf{w}(t), \overline{\mathbf{w}}(t))}{g(\mathbf{w}(t), \overline{\mathbf{w}}(t))},
$$

for $j=1, \ldots, n$. Consider the weight vector $P=\left(p_{1}, \ldots, p_{n}\right)$ and the face function $f_{P}$. Then $g_{P}=\varphi^{*} f_{P}$. By (30) we get the equalities:

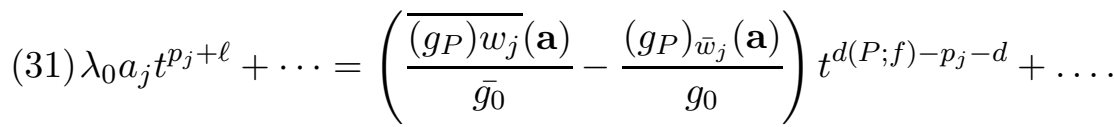

where $j=1, \ldots, n$ and $\mathbf{a}=\left(a_{1}, \ldots, a_{n}\right)$. The order of the left side for $j$ is $p_{j}+\ell$. The order of the right side is at least $d(P ; f)-p_{j}-d$. Thus we have

$$
p_{j}+\ell \geq d(P ; f)-p_{j}-d \text {. }
$$

Put

$$
C_{j}:=\left(\frac{\overline{\left(g_{P}\right)_{w_{j}}}(\mathbf{a})}{\overline{g_{0}}}-\frac{\left(g_{P}\right)_{\bar{w}_{j}}(\mathbf{a})}{g_{0}}\right) .
$$

The equality in (32) holds if $C_{j} \neq 0$ :

$$
p_{j}+\ell=d(P, g)-p_{j}-d, \quad \text { if } C_{j} \neq 0 .
$$

We assert that

Assertion 24. There exists some $j$ such that $C_{j} \neq 0$.

Proof. Assume that $C_{1}=\cdots=C_{n}=0$. This implies that $\overline{\nabla_{\partial} g(\mathbf{a})}=$ $u \nabla_{\bar{\partial}} g(\mathbf{a})$ with $u=\bar{b} / b$ and therefore we see that $\mathbf{a}$ is a critical point of $g_{P}: \mathbb{C}^{* n} \rightarrow \mathbb{C}$ by Proposition 1 ([] $)$ which contradicts to the non-degeneracy assumption.

Let $p_{\min }=\min \left\{p_{j} \mid j=1, \ldots, n\right\}$ and $J=\left\{j \mid p_{j}=p_{\min }\right\}$ and let $p_{\max }=$ $\max \left\{p_{j} \mid C_{j} \neq 0\right\}$ and $J^{\prime}=\left\{j \mid p_{j}=p_{\max }, C_{j} \neq 0\right\}$. We assert that

Assertion 25. $p_{\min }=p_{\max }$. 
Proof. Assume that $p_{\min }<p_{\max }$. Then we have a contradiction: For $k \in J$ and $j \in J^{\prime}$,

$$
p_{k}+\ell<p_{j}+\ell=d(P, g)-p_{j}-d<d(P, g)-p_{k}-d
$$

which contradicts to (33).

Thus we have proved the equivalence $C_{j}=0 \Longleftrightarrow j \notin J$ and comparing the leading coefficients of (31),

$$
\lambda_{0} a_{k}=C_{k}, p_{\min }+\ell=d(P, g)-p_{\text {min }}-d, \forall k \in J .
$$

Then taking the summation $\sum_{j \in J} p_{j} \bar{a}_{j} \times(34)$, we get the equality

$$
\sum_{k \in J} p_{k} \lambda_{0} \bar{a}_{k} a_{k}=\sum_{k \in J} p_{k} \bar{a}_{k} C_{k} .
$$

The left side is $\lambda_{0} \sum_{k \in J} p_{k}\left|a_{k}\right|^{2} \neq 0$. The right side is

$$
\begin{aligned}
\sum_{k \in J} p_{k} \bar{a}_{k} C_{k}= & \sum_{k=1}^{n} p_{k} \bar{a}_{k} C_{k} \\
= & \sum_{k=1}^{n} p_{k} \bar{a}_{k}\left(\overline{\left(g_{P}\right)_{w_{j}}}(\mathbf{a}, \overline{\mathbf{a}}) / \overline{g_{0}}-\left(g_{P}\right)_{\bar{w}_{j}}(\mathbf{a}, \overline{\mathbf{a}}) / g_{0}\right) \\
= & \left(\operatorname{rdeg}\left(P, g_{P}\right)+\operatorname{pdeg}\left(P, g_{P}\right)\right) \overline{g_{P}}(\mathbf{a}, \overline{\mathbf{a}}) / \overline{g_{0}} \\
& -\left(\operatorname{rdeg}\left(P, g_{P}\right)-\operatorname{pdeg}\left(P, g_{P}\right)\right) g_{P}(\mathbf{a}, \overline{\mathbf{a}}) / g_{0} .
\end{aligned}
$$

As the left side is non-zero, we have $g_{P}(\mathbf{a}, \overline{\mathbf{a}}) \neq 0$ and $d=d(P, g)$ and $g_{0}=g_{P}(\mathbf{a}, \overline{\mathbf{a}})$. Thus we finally obtain the equality

$$
\lambda_{0} \sum_{k \in J} p_{k}\left|a_{k}\right|^{2}=2 \operatorname{pdeg}\left(P, g_{P}\right)
$$

which implies $\lambda_{0}>0$ and thus $\lim _{t \rightarrow 0} \arg \lambda(t)=0$. As $d \theta(R(\mathbf{w}(t)))=$ $\Re \nabla \theta(R(\mathbf{w}(t)))=\Re \lambda(t)\|R(\mathbf{w}(t))\|^{2}>0$ for a sufficiently small $t$, this is a contradiction.

Remark 26. 1. Lemma 23 hold for any convenient non-degenerate mixed function $g(\mathbf{w}, \overline{\mathbf{w}})$ of strongly polar weighted homogeneous face type, as the proof do not use the assumption $g=\varphi^{*} f$.

2. Let $g=\varphi^{*} f$ where $f(\mathbf{z})$ is a convenient non-degenerate holomorphic function and $\varphi_{a, b}$ is a homogeneous cyclic covering map. Then

Assertion 27. The link topology of $g^{-1}(0)$ is a combinatorial invariant and it is determined by $\Gamma(f)$.

Proof. Assume that $f^{\prime}(\mathbf{z})$ is another convenient non-degenerate holomorphic function with $\Gamma\left(f^{\prime}\right)=\Gamma(f)$ and letg $^{\prime}=\varphi^{*} f^{\prime}$. Take a one-parameter family $f_{t}(\mathbf{z}), 0 \leq t \leq 1$ so that $\Gamma\left(f_{t}\right)=\Gamma(f), f_{0}=f, f_{1}=f^{\prime}$ and $f_{t}(\mathbf{z})$ is nondegenerate for any $t$. The we get a one-parameter family $g_{t}:=\varphi^{*} f_{t}$ of mixed function of strongly polar weighted homogeneous face type. Then their links are certainly isotopic. 
Observe that there exists in general mixed functions $h(\mathbf{w}, \overline{\mathbf{w}})$ which is convenient, non-degenerate and of strongly polar weighted homogeneous face type but it is not a homogenous lift of a holomorphic function. In such a case, the topology of the links of $g$ and $h$ may be different. See Example 5.4 in [7.

\section{REFERENCES}

[1] R. Berndt. An introduction to symplectic geometry, volume 26 of Graduate Studies in Mathematics. American Mathematical Society, Providence, RI, 2001. Translated from the 1998 German original by Michael Klucznik.

[2] C. Caubel, A. Némethi, and P. Popescu-Pampu. Milnor open books and Milnor fillable contact 3-manifolds. Topology, 45(3):673-689, 2006.

[3] H. Geiges. An introduction to contact topology, volume 109 of Cambridge Studies in Advanced Mathematics. Cambridge University Press, Cambridge, 2008.

[4] E. Giroux. Contact structures and symplectic fibrations over the circle.

[5] H. Hamm. Lokale topologische Eigenschaften komplexer Räume. Math. Ann., 191:235$252,1971$.

[6] J. Milnor. Singular points of complex hypersurfaces. Annals of Mathematics Studies, No. 61. Princeton University Press, Princeton, N.J., 1968.

[7] M. Oka. Mixed functions of strongly polar weighted homogeneous face type, arxiv $1202.2166 \mathrm{v} 1$.

[8] M. Oka. Topology of polar weighted homogeneous hypersurfaces. Kodai Math. J., 31(2):163-182, 2008.

[9] M. Oka. Non-degenerate mixed functions. Kodai Math. J., 33(1):1-62, 2010.

Department of Mathematics

TOKYO UNIVERSITY OF SCIENCE

1-3 Kagurazaka, Shinjuku-Ku

TOKYO 162-8601

E-mail: oka@rs.kagu.tus.ac.jp 